\title{
Hyperspherical entanglement entropy
}

\author{
J.S.Dowker ${ }^{1}$ \\ Theory Group, \\ School of Physics and Astronomy, \\ The University of Manchester, \\ Manchester, England
}

\begin{abstract}
The coefficient of the log term in the entanglement entropy associated with hyperspherical surfaces in flat space-time is shown to equal the conformal anomaly by conformally transforming Euclideanised spacetime to a sphere and using already existing formulae for the relevant heat-kernel coefficients after cyclic factoring. The result follows from the fact that the conformal anomaly on this lune has an extremum at the ordinary sphere limit. A proof is given.

Agreement with a recent evaluation of the coefficient is found.
\end{abstract}

1dowker@man.ac.uk 


\section{Introduction.}

In a recent work, Casini and Huerta, [1], have calculated the logarithmic term in the entanglement entropy of a conformally invariant scalar field associated with a $(d-2)$ spherical spatial subspace, $\Sigma$, in a flat space-time of any dimension, $d$. To be more precise, this entropy can be generically expanded in a short distance (high energy) cutoff $\epsilon$,

$$
S_{\Sigma}=\frac{g_{0}}{\epsilon^{d-2}}+\frac{g_{1 / 2}}{\epsilon^{d-3}}+\ldots+\frac{g_{(d-1) / 2}}{\epsilon}+g_{d / 2} \ln \epsilon+S_{0}
$$

where $S_{0}$ is the finite part and some of the $g_{i}$ coefficients may be zero. The logarithmic coefficient, $g_{d / 2}$, is universal and I would like in this short paper to outline an alternative derivation of the numerical results presented in [1]. The method used in [1] is a differential equation one and involves a transformation to a form for which

the heat-kernel can be derived simply. My method also involves heat-kernels and amounts effectively to an assembly of known results.

I give no discussion of the significance or use of the notion of entanglement entropy, and just refer to the useful review [2].

\section{General technique.}

I work with Euclideanised space-time which I compactify, metrically, to the $d$-sphere, $\mathrm{S}^{d}$. Because of conformal invariance, the coefficients $g_{d / 2}$ calculated in this case will be the same as the ones computed in [1] and by Solodukhin [3], for $d=4$. A purely numerical evaluation is given by Lohmayer et al, [4].

Solodukhin, [3], uses the holographic interpretation of entanglement entropy due to Ryu and Takayanagi, [5] to fix a coefficient and obtain the log coefficient for a generic conformal field, such as the scalar one used here. The value of the coefficient in four dimensions can also be found from the results of [5] for an $N=4$ superconformal field theory.

The general method for computing the entanglement entropy goes back to the early days of the topic, [6]. A conical singularity, of angle $2 \pi / B$, is introduced at the surface $\Sigma$ turning it into $\Sigma_{B}$. Space-time, $\mathcal{M}$, is thereby deformed to $\mathcal{M}_{B}$. The calculational formula is

$$
S_{\Sigma}=-\left.\left(1+B \partial_{B}\right) W\left(\mathcal{M}_{B}\right)\right|_{B=1}
$$


where $W$ is the effective action of the field theory. It has the expansion

$$
W\left(\mathcal{M}_{B}\right)=\frac{A_{0}}{\epsilon^{d-2}}+\frac{A_{1 / 2}}{\epsilon^{d-3}}+\ldots+\frac{A_{(d-1) / 2}}{\epsilon}+A_{d / 2} \ln (\epsilon)+W_{0}
$$

in terms of the heat-kernel short-time expansion coefficients, $A_{n}$, which depend on the geometry of $\mathcal{M}_{B}$ and, in particular, on $B$.

When $B=1$ space-time is smooth and the coefficients are the standard ones, discussed at length in the literature and can be referred to as the bulk coefficients. When $B \neq 1$, they take additional contributions from the singular codimension-2 submanifold, $\Sigma_{B}$, e.g. $[7,8]$,

$$
\begin{aligned}
A_{n}\left(\mathcal{M}_{B}\right) & =\left.\frac{1}{B} A_{n}\left(\mathcal{M}_{B}\right)\right|_{B=1}+A_{n}^{\Sigma_{B}}+O(1-B)^{2} \\
& =\frac{1}{B} A_{n}^{\text {bulk }}(\mathcal{M})+A_{n}^{\Sigma_{B}}+O(1-B)^{2}
\end{aligned}
$$

where the singular part, $A_{n}^{\Sigma_{B}}$ vanishes when $B=1$.

In the case that $\Sigma_{B}$ is a fixed point set under a global $\mathrm{O}(2)$ (for codimension2 ) isometry, there is no correction term in (4) since the $1 / B$ is an exact volume factor due to the isometry, [9-12]. In any case, such terms do not contribute to the entanglement entropy, (2).

If (4) is put into the expansion (3) and the entropy computed using (2) there results for the coefficient of the $\log \epsilon$ term the standard formula,

$$
g_{d / 2}=-\left.\partial_{B} A_{d / 2}^{\Sigma_{B}}\right|_{B=1}
$$

Actually $A_{n}^{\Sigma_{B}}$ always has the factor $(1-B)$, but I do not need this general fact. It emerges from the calculation to follow. As is well known, the coefficient $A_{d / 2}$ controls the conformal anomaly and is conformally invariant, [13].

\section{The $d$-sphere.}

As mentioned above, I choose for $\mathcal{M}$, the $d$-sphere and for its singular 'deformation', $\mathcal{M}_{B}$, the cyclic factor orbifold, $\mathrm{S}^{d} / \mathbb{Z}_{q}$. Then $B=q, q \in \mathbb{Z}$. The fixed point set is a $(d-2)$ dimensional sphere, $\mathrm{S}^{d-2}$. See also Fursaev and Miele, [14], Fujita, Nishioka and Takayanagi, [15]. 
Since all significant quantities are essentially polynomial in $q$, it is possible to analytically continue in $q$ in order to compute $g_{d / 2}$ from (5). This has been employed before, [16], again in connection with the $d$-lune, $\mathrm{S}^{d} / \mathbb{Z}_{q}{ }^{2}$

I have used this special case previously in [17] in order to fix the coefficients in the general form of the coefficient $A_{2}\left(\mathcal{M}_{B}\right)$ and regain Fursaev's result, [9], obtained using differential geometry. ${ }^{3}$ This would be sufficient to determine the log coefficient for $d=4$ but I wish to pursue any $d$ in this paper.

The essential calculation has been performed in [18] where the scalar heatkernel coefficients on $\mathrm{S}^{d} / \mathbb{Z}_{q}$ have been given for the case of conformal coupling in $d+1$ dimensions (i.e. $\xi R=(d-1) R / 4 d)$. For present purposes I need conformal coupling in $d$ dimensions (i.e. $\xi R=(d-2) R / 4(d-1))$. Because $R=d(d-1)$ is constant, a simple combination yields the required values.

\section{The calculation.}

The heat-kernel coefficients for conformal coupling in $d+1$ dimensions on the periodic $d$-lune are, [18] eqns (76), (77) (corrected with a factor of 1/2),

$$
\begin{aligned}
C_{d / 2}(q) & =\frac{2}{q d !} B_{d}^{(d)}((d-1) / 2 \mid \mathbf{d}) \\
C_{(d-k) / 2}(q) & =\frac{\Gamma(k / 2)}{q(k-1) !(d-k) !} B_{d-k}^{(d)}((d-1) / 2 \mid \mathbf{d}), k=1 \ldots d .
\end{aligned}
$$

A small technical point is that the printed formulae in [18] are for the lune of angle $\pi / q$ with Dirichlet and Neumann boundary conditions (corresponding to the \pm signs) on the $\mathrm{S}^{d-1}$ edges. In this case the size, $|\Gamma|$, of the polytope group is $2 q$ since reflections are included in $\Gamma$. To obtain the periodic lune the $D$ and $N$ values are added. In the usual fashion the angle of the lune is then doubled to $2 \pi / q$ corresponding to the pure rotation action of $\mathbb{Z}_{q}$. Relatedly, one confirms that there are no 'half-integer coefficients', as expected for a boundaryless manifold. Further, if $d$ is odd, the heat-kernel terminates with the $C_{(d-1) / 2}$ term.

In equation (6), the $B_{n}^{(d)}(x \mid \mathbf{d})$ are generalised Bernoulli polynomials and $\mathbf{d}$ is a $d$-dimensional vector of the degrees associated with the polytope group, $\Gamma$. In the present case all degrees, bar one, are unity, $\mathbf{d}=(q, 1, \ldots, 1) \equiv(q, \mathbf{1})$.

2 The lune of any angle can be treated directly. The case when $q=1 / n, n \in \mathbb{Z}$, corresponds to an $n$-copy covering of the sphere.

${ }^{3}$ Conformal invariance was employed in [17] to introduce extrinsic curvature terms. This method was taken further by Solodukhin, [3] in the present context and was also used extensively in [16]. 
I now set the coefficients $A_{k}$ in (3) equal to the coefficients, $\bar{C}_{k}(q)$, for conformal coupling in $d$ dimensions which, on noting that

$$
R\left(\frac{d-1}{4 d}-\frac{d-2}{4(d-1)}\right)=\frac{1}{4}
$$

are obtained as the combination,

$$
\bar{C}_{k}(q)=\sum_{l=0}^{k} \frac{1}{4^{k-l}(k-l) !} C_{l}(q), \quad k \in \mathbb{Z} .
$$

In particular, the total anomaly is (an alternative procedure is given in the Appendix),

$$
\bar{C}_{d / 2}(q)=\sum_{l=0}^{d / 2} \frac{1}{4^{d / 2-l}(d / 2-l) !} C_{l}(q),
$$

which is, of course, zero if the space-time dimension, $d$, is odd. As a consequence, the log coefficient trivially vanishes in this case.

The task now is to evaluate the generalised Bernoulli polynomials for even $d$, substitute into (9) and evaluate the log coefficient from (5) by

$$
g_{d / 2}=-\left.\partial_{q}\left(\bar{C}_{d / 2}(q)-\frac{1}{q} \bar{C}_{d / 2}(1)\right)\right|_{q=1}
$$

where the second term removes the bulk quantity.

The formulae needed to calculate the Bernoulli polynomials can be found in Nörlund [19]. Especially one finds the polynomial,

$$
B_{\nu}^{(n)}(x \mid q, \mathbf{1})=\sum_{s=0}^{\nu} q^{s}\left(\begin{array}{l}
\nu \\
s
\end{array}\right) B_{s} B_{\nu-s}^{(n-1)}(x),
$$

where $B_{\nu}^{(n-1)}(x) \equiv B_{\nu}^{(n-1)}(x \mid \mathbf{1}) \equiv B_{\nu}^{(n-1)}(x \mid 1, \ldots, 1)$ is the commonly occurring Bernoulli polynomial whose degrees are all unity and is given by the polynomial in $x$,

$$
B_{\nu}^{(n)}(x)=\sum_{s=0}^{n}\left(\begin{array}{l}
\nu \\
s
\end{array}\right) x^{s} B_{\nu-s}^{(n)} .
$$

Here $B_{\nu}^{(n)} \equiv B_{\nu}^{(n)}(0)$ are generalised Bernoulli numbers which can be calculated in various ways. A recursion formula is, [19] p.195 equn. (14),

$$
B_{\nu}^{(n)}=\sum_{s=1}^{\nu}(-1)^{s}\left(\begin{array}{l}
\nu \\
s
\end{array}\right) B_{s} B_{\nu-s}^{(n)}
$$


with $B_{0}^{(n)}=1$.

From (6) one requires the derivative at $q=1$,

$$
\left.\partial_{q} B_{\nu}^{(n)}(x \mid q, \mathbf{1})\right|_{q=1}=\sum_{s=0}^{\nu} s\left(\begin{array}{c}
\nu \\
s
\end{array}\right) B_{s} B_{\nu-s}^{(n-1)}(x)
$$

From these ingredients it can be shown ( $c f$ the Appendix) that the conformal anomaly on the lune has an extremum at $q=1$ so that, from (10), $g_{d / 2}$ equals the conformal anomaly at this point, i.e. on the ordinary $d$-sphere. This has been evaluated many times before and here follows very easily from (6) and (9). The numbers are those given in [1].

\section{Conclusion.}

The content of this brief note is strictly technical and just presents an alternative derivation of the universal coefficient of the log term in the entanglement entropy for hyperspheres. The full expression for the entropy will be discussed at another time ${ }^{4}$ as well as the relevance of the result for the holographic approach, $[3,5,20]$.

\section{Acknowledgements.}

I wish to thank Robert Myers for suggestions and information.

\section{Appendix.}

I prove that the conformal anomaly on $\mathrm{S}^{d} / \mathbb{Z}_{q}$ has an extremum at the ordinary sphere limit, $q=1$. For this purpose, it is convenient to use an alternative form for the anomaly obtained in [21]. From general theory, the coefficient, $\bar{C}_{d / 2}^{(d)}$ is the value of the relevant $\zeta$-function, $\bar{\zeta}(s)$, evaluated at $s=0$. This provides a more sophisticated approach.

\footnotetext{
${ }^{4}$ I will show that the effective action on odd dimensional lunes has an extremum at the ordinary sphere.
} 
It is shown in [21] that this value is given in terms of Barnes' $\zeta$-function, $\zeta_{d}$, by, in the present case, ${ }^{5}$

$$
\bar{\zeta}(0)=\frac{1}{2}\left(\zeta_{d}(0, a-\alpha \mid q, \mathbf{1})+\zeta_{d}(0, a+\alpha \mid q, \mathbf{1})\right),
$$

where $\alpha=1 / 2$ for conformal coupling in $d$ dimensions. (This corresponds to (7).) In this way I can bypass the particular construction (9). For Neumann conditions $a=(d-1) / 2$, and, for Dirichlet, $a=(d-1) / 2+q$.

Then

$$
\bar{C}_{d / 2}^{N, D}=\frac{1}{2 q d !}\left(B_{d}^{(d)}(d / 2-1 \mid q, \mathbf{1})+B_{d}^{(d)}(d / 2 \mid q, \mathbf{1})\right),
$$

valid for both Neumann and Dirichlet after using (for even $d$ ) the symmetry,

$$
B_{n}^{(d)}(d-1+q-x \mid q, \mathbf{1})=(-1)^{n} B_{n}^{(d)}(x \mid q, \mathbf{1}) .
$$

To differentiate with respect to $q$, which is one of the degrees (or parameters), I use the result of Barnes, [23] §13, for my situation,

$$
q \partial_{q} \frac{1}{q} B_{d}^{(d)}(x \mid q, \mathbf{1})=-\frac{1}{q} B_{d}^{(d+1)}(x+q \mid q, q, \mathbf{1}),
$$

so that, from (9), at $q=1$, with $\bar{C}=\bar{C}^{N}+\bar{C}^{D}$,

$$
\left.d ! \partial_{q} \bar{C}_{d / 2}\right|_{q=1}=-B_{d}^{(d+1)}(d / 2 \mid \mathbf{1})-B_{d}^{(d+1)}(d / 2+1 \mid \mathbf{1}),
$$

each term of which vanishes in view of the product expression, (e.g. [19] p.186, [24], $\S 8)$,

$$
B_{d}^{(d+1)}(x \mid \mathbf{1})=(x-1)(x-2) \ldots(x-d),
$$

and the fact that $d$ is even. This proves the required result. ${ }^{6}$

Furthermore, equation (11) provides for a simpler computation of the anomaly itself. The equivalence with (9) corresponds to a Bernoulli identity which I do not need to elaborate.

\footnotetext{
5 The two terms reflect the factorisation of the eigenvalues into two factors. For the significance of this result see Allen [22], and [21]. There is no multiplicative anomaly for the conformal anomaly.

${ }^{6}$ Further work is needed to exclude a point of inflection. This can be done by finding the second derivative by continuing the process indicated in (12). It alternates in sign as $(-1)^{d / 2}$, as does the conformal anomaly.
} 


\section{References.}

1. Casini,H. and Huerta,M. Entanglement entropy for the $n$-sphere, arXiv:1007.1813.

2. Casini,H. and Huerta,M. J. Phys. A42 (2009) 504007.

3. Solodukhin,S.N. Phys. Letts. B665 (2008) 305.

4. Lohmayer,R., Neuberger,H, Schwimmer,A. and Theisen,S. Numerical determination of entanglement entropy for a sphere, ArXiv:0911.4238.

5. Ryu,S. and Takayanagi,T. JHEP 0608(2006)045.

6. Callan,C.G. and Wilczek,F. Phys. Letts. B333 (1994) 55.

7. Fursaev,D.V. and Solodukhin,S.N. Phys. Letts. B365 (1996) 51.

8. Fursaev,D.V. and Solodukhin,S.N. Phys. Rev. D52 (1995) 2133.

9. Fursaev,D.V. Phys. Letts. B334 (1994) 53.

10. Dowker,J.S.Phys. Rev. D50 (1994) 6369.

11. Donnelly,H. Math. Ann. 224 (1976) 161.

12. Donnelly.H. Indiana U. Math.J. 27 (1978) 889.

13. Dowker,J.S. and Kennedy,G. J. Phys. A (1978) 895.

14. Fursaev,D.V. and Miele,G. Phys. Rev. D49 (1994) 987.

15. Fujita,M.,Nishioka,T. and Takayanagi,T. JHEP 0809 (2008) 016.

16. Apps,J.S. and Dowker,J.S. Class. Quant. Grav. 15 (1998) 1121.

17. Dowker,J.S.Class. Quant. Grav. 11 (1994) L137.

18. Chang,P. and Dowker,J.S. Nucl. Phys. B395 (1993) 407.

19. Nörlund,N.E. Acta Mathematica 43 (1922) 121.

20. Myers,R.C. and Sinha, A. Seeing a c-theorem with holography, ArXiv:1006.1263

21. Dowker,J.S. Comm. Math. Phys. 162 (1994) 633.

22. Allen,B. PhD Thesis, University of Cambridge, 1984.

23. Barnes,E.W. Trans. Camb. Phil. Soc. 19 (1903) 374.

24. Barnes,E.W. Trans. Camb. Phil. Soc. 19 (1903) 426. 